\title{
A Rare Combination of Posterior Elbow Dislocation with Ipsilateral Intra- Articular Distal Radius Fracture with Radiocarpal Dislocation: Case Report and Literature Review
}

\author{
Dany K. Aouad Ramzi Musharrafieh Fouad Jabbour Nabil Dib \\ Alexandre H. Nehme \\ Department of Orthopedic Surgery, Saint George Hospital Medical Center, University of \\ Balamand, Beirut, Lebanon
}

Keywords

Elbow dislocation $\cdot$ Distal radius fracture $\cdot$ Ipsilateral

\section{Abstract}

Separate elbow dislocation and forearm fractures are common injuries, with both injuries occurring concomitantly and ipsilaterally being rare. We report a case of a 70-year-old female patient who had a posterior elbow dislocation with ipsilateral comminuted distal radius fracture with anterior radiocarpal dislocation. Closed reduction of the elbow was done with open reduction and internal fixation of the distal radius fracture. At 6-month follow-up, the patient had no pain, with satisfactory range of motion of both joints, resuming her previous daily activities.

\section{Introduction}

The second most commonly dislocated joint is the elbow. Posterior dislocations are more common than the anterior varieties, which are very rare. Two types of dislocations are described: simple dislocations without any associated fractures and complex ones associated with a fracture, the latter being found in $20 \%$ of the cases with associated proximal ulna and radius involvement $[1,2]$. Only few cases of posterior elbow dislocations with ipsilateral distal radius have been reported in the literature, mainly affecting young and pediatric populations with high-energy mechanism injuries [3-5]. To the best of our knowledge, no cases of 


\section{Case Reports in Orthopedic Research}

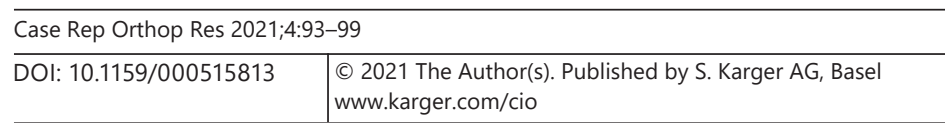

Aouad et al.: Posterior Elbow Dislocation with Complex Intra-Articular Distal Radius Fracture

Fig. 1. Diagram showing the mechanism of injury of the described pathology, consisting of compressive longitudinal force with valgus and external rotational stress on a semiflexed elbow causing dislocation with direct impact on a radially oriented flexed wrist resulting in an anteriorly displaced radial fracture with anterior radiocarpal dislocation and ulnar styloid avulsion fracture.

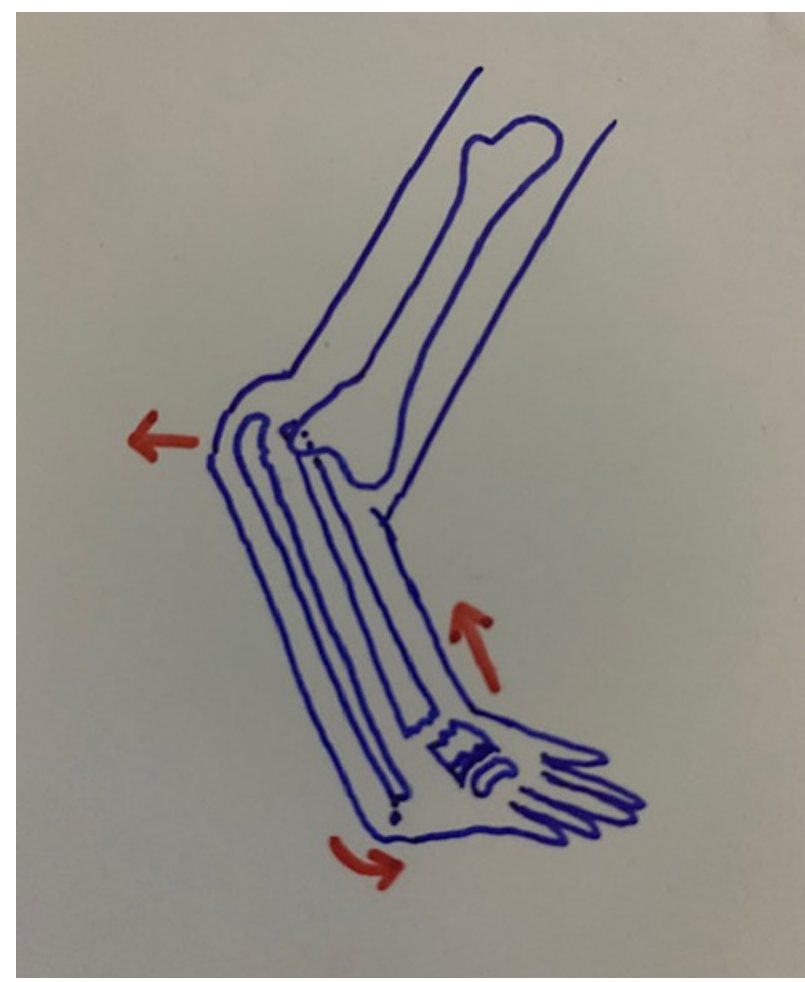

Barton fracture with associated ipsilateral posterior elbow dislocation have been previously reported. The purpose of this paper is to present the case of an elderly female patient with a closed posterior elbow dislocation associated with an ipsilateral intra-articular comminuted distal radius fracture with anterior radiocarpal dislocation.

\section{Case Presentation}

A 70-year-old female patient with a past medical history of hypertension and coronary artery disease presented to the emergency department after a fall from the height of her bed while turning during sleep. She reported hitting the ground on a flexed right wrist and semiflexed right elbow (Fig. 1). She complained of severe right elbow pain with inability of flexion, extension, as well as forearm pronation and supination. She also reported severe right wrist pain with very limited range of motion. On physical examination, both the patient's elbow and wrist were moderately swollen with gross deformation of the wrist with an extended elbow. Range of motion of both joints was painfully restricted, without any distal neurovascular deficit or abnormality. Plain radiographs were done showing a posterior elbow dislocation and a comminuted anteriorly displaced distal radius fracture with anterior radiocarpal dislocation and severe posterior comminution (Fig. 2, 3).

After adequate pain management and low-dose sedation in the emergency department setting, successful closed reduction of the elbow was done (Fig. 4), followed by arm sling immobilization and posterior cast placement over the distal radius fracture. After reduction, neurovascular exam is found to remain normal. This was followed by serial neurovascular exams throughout her stay at the emergency room, showing no acute changes with normal radial and ulnar pulses with associated minimal anterior elbow swelling. 


\section{Case Reports in Orthopedic Research}

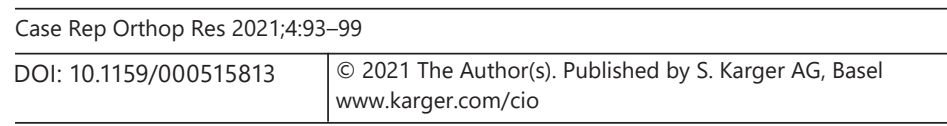

Aouad et al.: Posterior Elbow Dislocation with Complex Intra-Articular Distal Radius Fracture
Fig. 2. Plain radiographs of the right elbow anteroposterior and lateral views showing a posterior elbow dislocation with no visualized associated fractures.
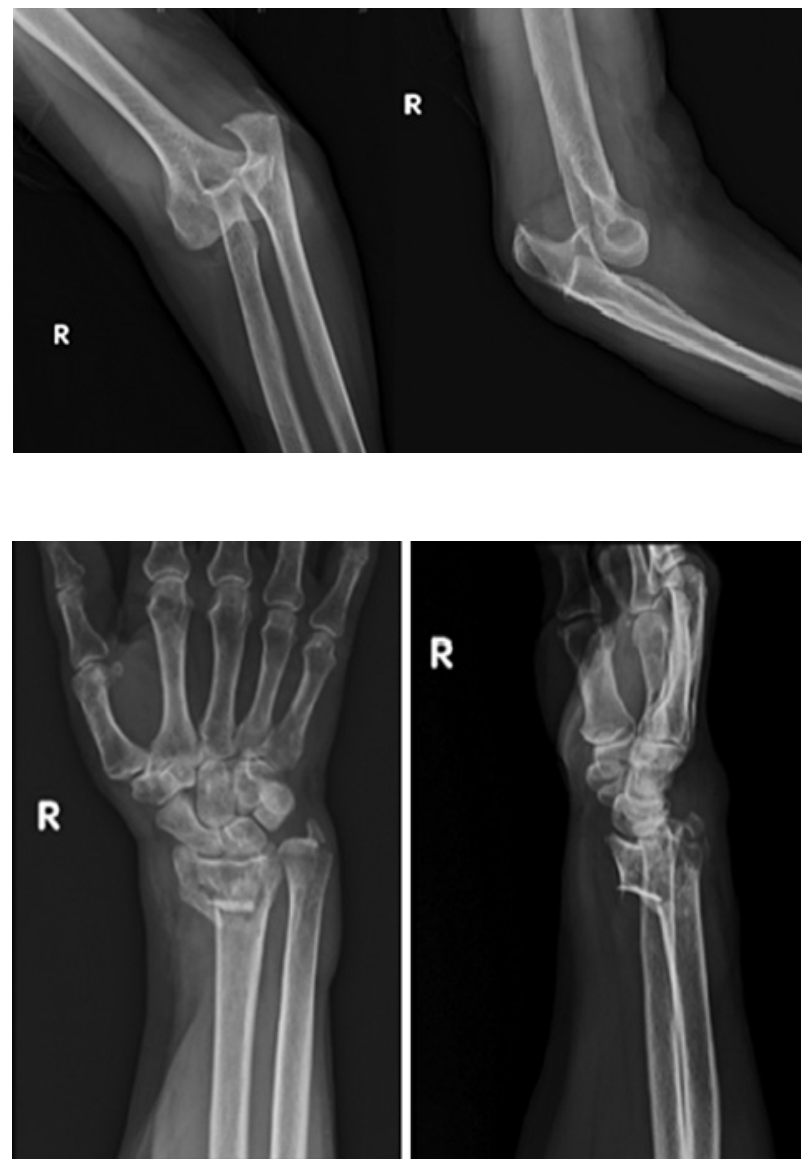

Fig. 3. Plain radiographs of the right wrist in the anteroposterior and lateral views showing a comminuted intra-articular anteriorly displaced distal radius fracture with anterior radiocarpal dislocation and severe posterior comminution.

Patient then underwent computed tomography imaging with 3D reconstuction for both the elbow and wrist for assessment of fracture pattern and distal radius fracture intra-articular assessment. CT scan showed a congruous right elbow joint with no fractures. It also showed a right distal radius comminuted intra-articular fracture with anterior radiocarpal dislocation (Fig. 5, 6).

She was then admitted to the ward for open reduction and internal fixation of her distal radius fracture. Under nerve block and sedation, manipulation of the elbow with valgus/ varus stress tests showed a stable joint. After scrubbing and draping, volar approach to the distal radius taken reaching the fracture site, trial of reduction maneuvers done followed by $\mathrm{K}$-wire fixation. Distal radius plate inserted under fluoroscopic control and fixation with ten screws. Due to severe comminution and small size, the posteriorly comminuted fragments were not candidates for K-wire fixation or screw purchase.

At 6 weeks follow-up, patient splint removed and extension wrist brace is placed. X-rays of the right wrist showed signs of bone healing over the previous fracture site, along with residual posterior wall comminution (Fig. 7b). X-ray of the right elbow showed good bony alignment with no signs of dislocation or fractures (Fig. 7b). Physical therapy was started, consisting of gentle passive and active-assisted wrist mobilization. Patient reports absence of symptoms such as pain, weakness, swelling, etc. Due to travelling causes, patient was lost to clinical follow-up; however, she was contacted by telephone and reported no residual symptoms with satisfactory range of motion and return to previous activities at 6 months postoperatively. She was also instructed to undergo bone densitometry to assess bone stock and any possible osteoporosis requiring treatment. 


\section{Case Reports in Orthopedic Research}

\begin{tabular}{l|l}
\hline Case Rep Orthop Res 2021;4:93-99 \\
\hline DOI: 10.1159/000515813 & $\begin{array}{l}\text { @ 2021 The Author(s). Published by S. Karger AG, Basel } \\
\text { www.karger.com/cio }\end{array}$ \\
\hline
\end{tabular}

Aouad et al.: Posterior Elbow Dislocation with Complex Intra-Articular Distal Radius Fracture

Fig. 4. Post closed reduction plain radiograph of the right elbow in the lateral view showing successful reduction with no visualized associated fractures.
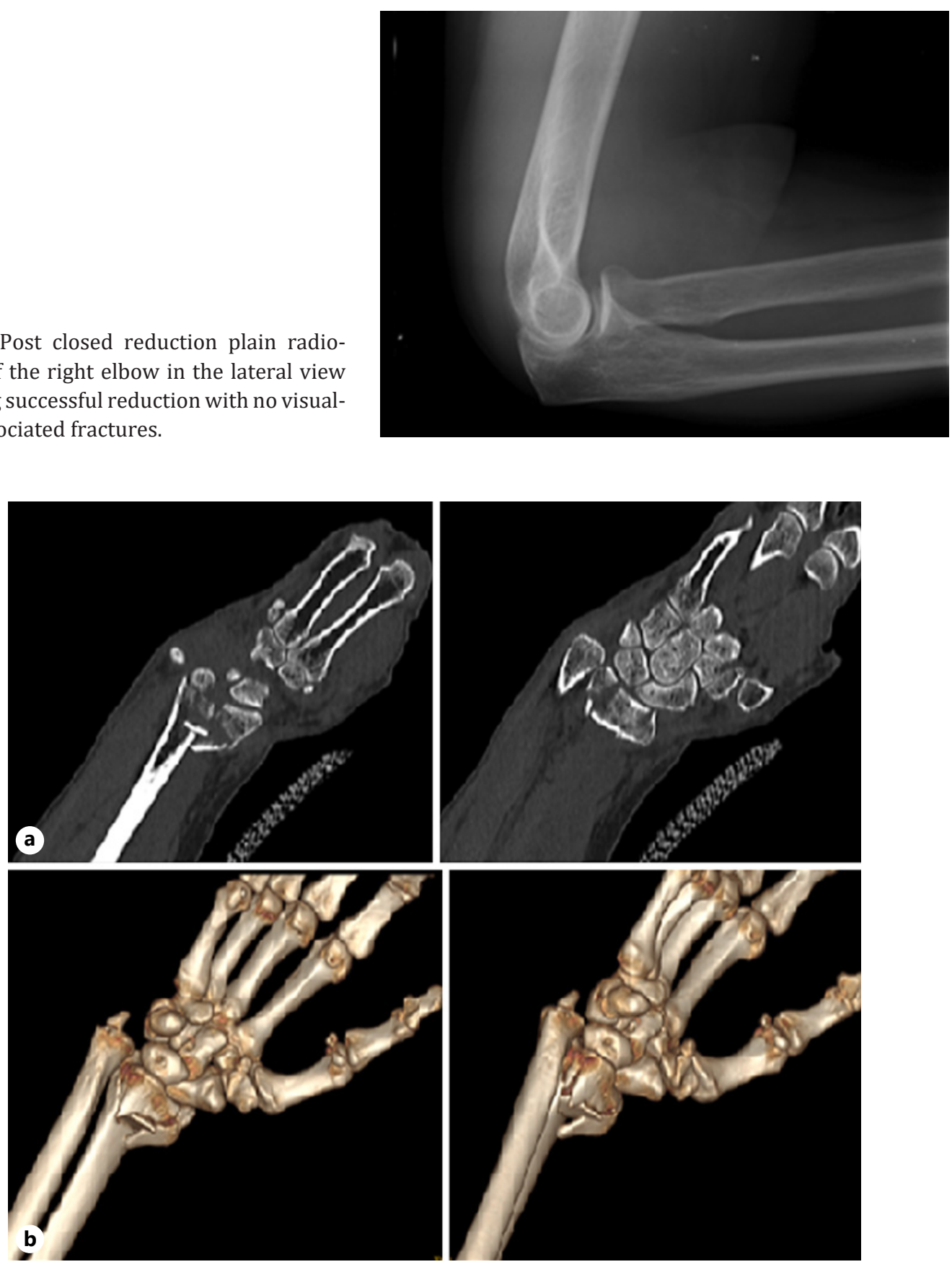

Fig. 5. Computed tomography imaging cuts of the right wrist showing (a) in the coronal views (b) and 3D reconstruction views a comminuted intra-articular displaced and fragmented fracture of the distal radius with associated ulnar styloid fracture and anteriorly dislocated radiocarpal joint.

\section{Discussion}

The elbow is a hinged joint and is one of the most stable articulations. The most commonly associated fractures with elbow dislocation are radial head, coronoid process, and olecranon fractures [2,6]. Elbow dislocations with concomitant ipsilateral ulnar and radial diaphyseal fractures have been reported [7]. Elbow dislocation with ipsilateral distal radius fracture is a 
Fig. 6. Computed tomography of the right elbow postreduction in the sagittal and 3D reconstruction views showing successful reduction with no associated fractures.
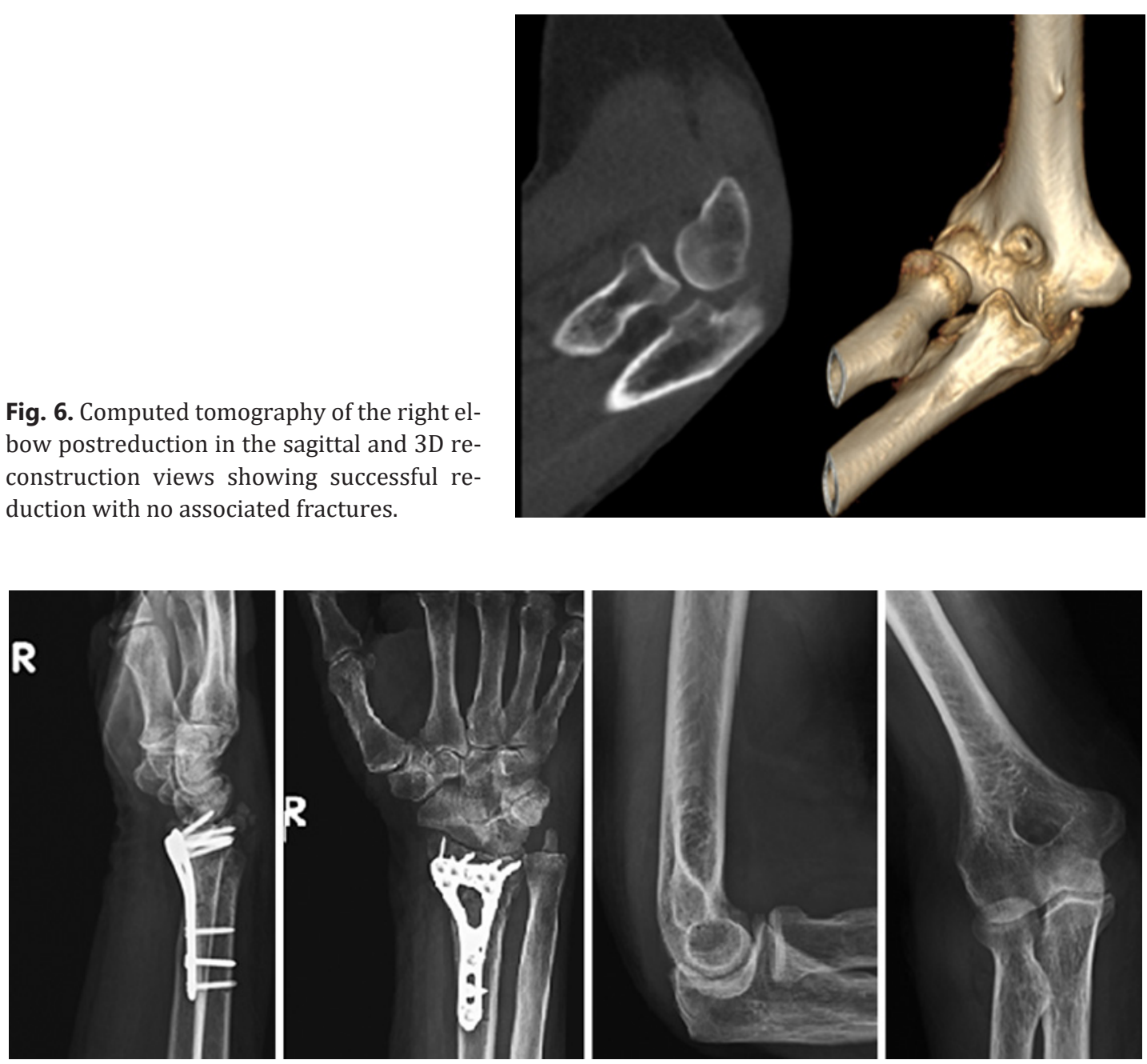

Fig. 7. a Plain radiograph in the anteroposterior and lateral views of the right wrist showing a distal radius plate and ten screws with signs of bone healing over the previous fracture site, along with residual posterior wall comminution. b Anteroposterior and lateral views X-ray of the right elbow showing good bony alignment with no signs of dislocation or fractures.

rare pattern of injury, with most reported cases being either compound fracture dislocations or affecting the pediatric population [1]. The association of a comminuted intra-articular distal radius fracture with anterior radiocarpal dislocation and an ipsilateral posterior elbow dislocation has not been reported in the literature. The probable mechanism of injury is speculated to be a compressive longitudinal force with valgus and external rotational stress on a semi-flexed elbow causing dislocation with direct impact on a radially oriented flexed wrist resulting in an anteriorly displaced radial fracture with anterior radiocarpal dislocation and ulnar styloid avulsion fracture (Fig. 7).

Lahoz et al. [8] reported 3 cases of elbow dislocation with ipsilateral fracture of the distal radius with a brachial artery injury, stressing on the importance of vascular assessment in such cases, with high suspicion of brachial artery injuries requiring urgent postreduction surgical repair [8]. In the presented case, postoperatively, the patient's elbow and wrist were immobilized with a posterior cast for one week, after which a hinged elbow brace with a wrist posterior cast was placed. Early mobilization and physical therapy were started in order to prevent elbow stiffness and decrease the risk of algodystrophy for which she is at increased 
Case Reports

in Orthopedic Research

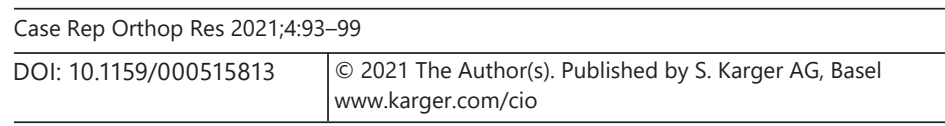

Aouad et al.: Posterior Elbow Dislocation with Complex Intra-Articular Distal Radius Fracture

risk due to her combined injuries and age. Six months postoperatively and after adequate rehabilitation, the patient was able to resume her previous activities with no residual pain or limitations.

\section{Conclusion}

Elbow dislocation with ipsilateral distal radius fracture is a rare pathology, especially one with intra-articular and radiocarpal joint involvement. It is of great importance to assess both elbow and wrist joints when such patterns are suspected, mainly if the mechanism of injury is such as the one described above. Neurovascular assessment is imperative, focusing on the integrity of the brachial artery, mostly in posterior elbow dislocations. Last but not least, it is imperative to assess the above and below joint structures in trauma patients for possible concomitant injuries.

\section{Statement of Ethics}

Approval and consent of the Ethics Committee have been received for the publication of this article. Written informed consent was obtained from the patient for publication of this case report AND any accompanying images.

\section{Conflict of Interest Statement}

The authors declare that they have no competing interests.

\section{Funding Sources}

No funds were received in support of this study.

\section{Author Contributions}

D.A.: writing and editing the article, with editing of the images and radiographs. R.M.: writing and editing the article. F.J.: writing and editing the article. N.D.: writing and editing the article. A.N.: Surgeon who supervised writing and finalizing the article.

\section{Data Availability Statement}

The datasets used and/or analyzed during the current study are available from the corresponding author on reasonable request.

\section{Karger'}




\section{References}

1 Meena S, Trikha V, Kumar R, Saini P, Sambharia AK. Elbow dislocation with ipsilateral distal radius fracture. J Nat Sci Biol Med. 2013;4(2):479-81.

2 Ring D, Jupiter JB. Fracture-dislocation of the elbow. J Bone Joint Surg Am. 1998;80(4):566-80.

3 Batra S, Andrew JG. Ipsilateral compound distal radius fracture with missed elbow dislocation. A rare injury pattern. Eur J Emerg Med. 2007;14(6):363-4.

4 Soon JC, Kumar VP, Satkunanartham K. Elbow dislocation with ipsilateral radial shaft fracture. An unusual outcome. Clin Orthop Relat Res. 1996;329(329):212-5.

5 Nanno M, Sawaizumi T, Ito H. Transverse divergent dislocation of the elbow with ipsilateral distal radius fracture in a child. J Orthop Trauma. 2007;21(2):145-9.

6 Mezera K, Hotchkiss RN. In: Bucholz RW, Heckman JD, Court-Brown CM, editors. Fractures and dislocations of the elbow. Rockwood and Green's fractures in adults, Vol. 1. Philadelphia: Lippincott Williams \& Wilkins; 2001. p. 921-52.

7 Hung SC, Huang CK, Chiang CC, Chen TH, Chen WM, Lo WH. Monteggia type 1 equivalent lesion: diaphyseal ulna and radius fractures with a posterior elbow dislocation in an adult. Arch Orthop Trauma Surg. 2003;123: 311-3.

8 Lahoz LT, Gomez CL, Reiriz JS, Rodriguez JD, Renart IP. Elbow dislocation with ipsilateral fracture of the distal radius associated with a brachial artery injury: a new pathological condition of traumatic origin. Rev Esp Cir Ortop Traumatol. 2017;61(3):146-53. 